OPEN ACCESS

Edited by:

Zahid Hussain,

University of Sharjah, United Arab Emirates

Reviewed by:

Hnin Ei Thu,

National University of Malaysia,

Malaysia

Rai Sarfraz,

University of Sargodha, Pakistan

Shahzeb Khan,

University of Texas at Austin,

United States

*Correspondence:

Ju-Yen Fu

fujuyen@mpob.gov.my

Lay-Hong Chuah

alice.chuah@monash.edu

Specialty section:

This article was submitted to

Experimental Pharmacology and Drug

Discovery,

a section of the journa

Frontiers in Pharmacology

Received: 16 September 2021

Accepted: 10 December 2021

Published: 07 January 2022

Citation:

De Silva L, Fu J-Y, Htar TT,

Wan Kamal WHB, Kasbollah A,

Muniyandy S and Chuah L-H (2022)

Biodistribution Study of Niosomes in

Tumor-Implanted BALB/C Mice Using

Scintigraphic Imaging.

Front. Pharmacol. 12:778396.

doi: 10.3389/fphar.2021.778396

\section{Biodistribution Study of Niosomes in Tumor-Implanted BALB/C Mice Using Scintigraphic Imaging}

\author{
Leanne De Silva ${ }^{1}$, Ju-Yen Fu ${ }^{2 *}$, Thet Thet Htar ${ }^{1}$, Wan Hamirul Bahrin Wan Kamal ${ }^{3}$, \\ Azahari Kasbollah ${ }^{3}$, Saravanan Muniyandy ${ }^{4}$ and Lay-Hong Chuah ${ }^{1 *}$
}

${ }^{1}$ School of Pharmacy, Monash University Malaysia, Bandar Sunway, Malaysia, ${ }^{2}$ Nutrition Unit, Malaysian Palm Oil Board, Bandar Baru Bangi, Malaysia, ${ }^{3}$ Medical Technology Division, Malaysian Nuclear Agency, Bangi, Malaysia, ${ }^{4}$ Department of Pharmacy, Fatima College of Health Sciences, Al Ain, United Arab Emirates

The purpose of this work was to study the biodistribution of niosomes in tumor-implanted BALB/c mice using gamma scintigraphy. Niosomes were first formulated and characterized, then radiolabeled with Technetium-99 $\mathrm{m}\left({ }^{99 \mathrm{~m}} \mathrm{Tc}\right)$. The biodistribution of 99mTc-labeled niosomes was evaluated in tumor-bearing mice through intravenous injection and imaged with gamma scintigraphy. The labeled complexes possessed high radiolabeling efficiency $(98.08 \%)$ and were stable in vitro (>80\% after $8 \mathrm{~h}$ ). Scintigraphic imaging showed negligible accumulation in the stomach and thyroid, indicating minimal leaching of the radiolabel in vivo. Radioactivity was found mainly in the liver, spleen and kidneys. Tumor-to-muscle ratio indicated a higher specificity of the formulation for the tumor area. Overall, the formulated niosomes are stable both in vitro and in vivo, and show preferential tumor accumulation.

Keywords: biodistribution, nanotechnology, nanocarrier, niosomes, radiolabeling, gamma scintigraphy

\section{INTRODUCTION}

There has been a rapid growth of interest in the field of nanocarriers particularly for its application in cancer therapy in terms of imaging, diagnosis and treatment over the past 3 decades (Hafner et al., 2014; Choudhury et al., 2018; Gao et al., 2018; Hussain et al., 2021). Some commonly researched nanocarriers include polymeric nanospheres, polymeric micelles, dendrimers, gold nanoparticles, iron oxide nanoparticles, solid lipid carriers and vesicular systems such a liposomes, transferomes and niosomes. To date, there are close to 250 nanomedical products which have either been approved or are currently in the final stages of approval by the Food and Drug Administration (FDA) and European Medicines Agency (EMA) for the treatment of a variety of diseases including cancer (Etheridge et al., 2013; Goldman et al., 2017). Currently, the most widely used anti-cancer nanoformulations in the market are for breast cancer treatment sold under the tradenames, Doxil and Abraxane.

The strategy of developing a magic bullet drug against a wide range of solid tumors would be an impossible task due to the vast genetic diversification and mutations of cancer in different individuals (Björnmalm et al., 2017). The enhanced permeation and retention (EPR) effect was widely used in the development of nanocarriers for passive targeting (Subhan et al., 2021), where it involves the preferential accumulation of nanocarriers within solid tumors due to their leaky vessels that are formed as a result of uncontrolled angiogenesis (Baban et al., 1998; Byrne et al., 2008; Hatakeyama et al., 2011). Therefore, this brings about the postulation that systemically administered nanocarriers that are smaller in diameter than the fenestrations, but larger than the tight endothelial junctions, 
have the potential to gain access within the interstitium and be entrapped within the tumor microenvironment (He et al., 2020; Shi et al., 2020). Furthermore, this accumulation is further facilitated by the tumor's lack of well-defined lymphatic vessels that impairs extravasation of the nanocarrier out of the tumor (Haley et al., 2008; Hak et al., 2010).

Incomplete understanding on the nature of EPR has led to extensive debate in recent years. However, Maeda et al. (2021) suggested that the success or failure of EPR is highly dependent on the design of nanomedicine rather than the EPR itself. An important prerequisite for a nanocarrier is a reasonably long enough half-life to enable the EPR effect to take place. Additionally, another obstacle to effective drug delivery to the tumor microenvironment is the clearance by the mononuclear phagocyte system (MPS) (Allen and Cullis, 2013; Chapman et al., 2013). Upon entering the circulatory system, nanocarriers are often opsonized and taken up by phagocytic cells in the blood, spleen, liver and bone marrow, leaving only a small fraction of nanocarriers in the circulation that would be available for uptake at the tumor site (Chapman et al., 2013). This barrier was first overcome in the early 1990s, by another major breakthrough in the field of drug delivery with the invention of long circulating liposomes that was achieved by incorporating poly (ethylene glycol) (PEG) into the bilayer (Hatakeyama et al., 2011). This theory arose from the observation that hydrophobic particles tend to be opsonized more rapidly in the bloodstream due to the increased adsorption of blood serum proteins on their surfaces. Studies have since shown that the presence of PEG on the surface of nanoparticles can increase its blood circulation time. This is attributed to the increase in hydrophilicity, which enhances the stability of the bilayer while simultaneously introducing steric hindrance, resulting in decreased recognition by the MPS and inhibition of opsonization through protein adsorption (Hussain et al., 2019). Taken together, this results in prolonged biodistribution of the nanocarrier in the bloodstream (Allen et al., 1991; Haley et al., 2008; Hatakeyama et al., 2011; Muthu et al., 2011; Hussain et al., 2019). Another crucial factor in enabling EPR is the size of nanocarriers. It is often suggested that a size of $50-200 \mathrm{~nm}$ is preferred to fit tumor vascular fenestration (Nel et al., 2017) whereas sizes above $100 \mathrm{~nm}$ is preferred to escape renal clearance (Maeda 2021). Although low molecular weight compounds can similarly achieve passive targeting and show selective tumor uptake due to their size range, prolonged retention in the tumor can only be seen in nanomedicine (Maeda, 2021).

Niosomes are lipid bilayer nanocarriers which have previously been shown to promote tumor selective delivery of chemotherapeutics such as tamoxifen (Shaker et al., 2015) and vinblastine (Amiri et al., 2018). Niosomes fabricated in the nanometer range has been proven to be able to encapsulate hydrophobic and hydrophilic compounds (Maniam et al., 2021). In our previous study, niosomes prepared with Span 60 were presented with high biocompatibility and stability in human serum (De Silva et al., 2019). That being said, some suggested that vesicle nanocarriers like liposomes may not release encapsulated compounds even if they are delivered to the tumors via EPR effect if the liposomes are too stable (Maeda, 2021). To date, little is understood on the biodistribution of niosomes in relation to EPR mechanisms.

Determining the fate of a nanocarrier in vivo via biodistribution studies is a vital component in evaluating its potential safety and efficacy. Biodistribution studies typically involve the administration of a compound followed by quantifying the amount of the compound in various tissues and organs at different time-points (Patel et al., 2016). More recently, imaging techniques has attracted a lot of attention as they are less invasive and time consuming; at the same time being more cost effective in terms of quantity of animals and analytical reagents used (Ding et al., 2012; Patel et al., 2016). An example includes nuclear molecular imaging, which involves the imaging of molecules such as nanocarriers delivered to the body of a living multicellular organism. In this regard, scintigraphic imaging can be used for the tracking of gamma-emitting (photon) radionuclide compounds in vivo for diagnostic and therapeutic purposes (Boerman et al., 2000; Patel et al., 2016). The $\gamma$-ray emissions from the radionuclides can be detected by singlephoton emission computed tomography (SPECT) to construct an image or be used to estimate radiation dosimetry (Jackson et al., 2020; Zhang et al., 2020).

This study utilizes gamma scintigraphic imaging to track the movements of niosomes in mice. The niosomes will first be labeled with $99 \mathrm{mTc}$, then injected into tumor-bearing mice for tracking and imaging. The radioactivity in each organ indicates the presence of niosomes, which allows us to understand the biodistribution pattern and the fate of the niosomes in mice realtime.

\section{MATERIALS AND METHODS}

\subsection{Materials}

D-a-tocopherol polyethylene glycol 1,000 succinate (TPGS), 4dimethylaminopyridine (DMAP), diethylenetriaminepentaacetic acid (DTPA) anhydride, stannous chloride dihydrate $\left(\mathrm{SnCl}_{2} \cdot \mathrm{H}_{2} \mathrm{O}\right)$, Sorbitan monostearate (Span) 60, cholesterol, PBS, and pyridine were purchased from SigmaAldrich (St Louis, MO, United States). Dimethyl sulfoxide (DMSO) and $\mathrm{BALB} / \mathrm{c}$ mouse serum was obtained from Fisher Scientific (Novi, MI, United States). Dialysis tubing (molecular weight cut-off 1,000 Da) was purchased from Spectrum Labs (San Francisco, CA, United States). Sodium bicarbonate $\left(\mathrm{NaHCO}_{3}\right)$, sodium hydroxide $(\mathrm{NaOH})$, and hydrochloric acid $(\mathrm{HCl})$ were obtained from Systerms ChemAR (Shah Alam, Malaysia). Sephadex G-25 column was purchased from GE Healthcare Bio-Sciences Corp. (Piscataway, NJ, United States). Instant thin layer chromatography (ITLC) silica gel (SG)-coated fiber glass sheet was obtained from Agilent Technologies (Santa Clara, CA, United States). Drytec $99 \mathrm{mTc}$ generator ( ${ }^{99}$ Mo activity: $508.4 \mathrm{mCi}$ ) was purchased from GE Healthcare United Kingdom Ltd. (Little Chalfont, United Kingdom). Breast cancer 4T1 cells were obtained from ATCC, United States. Dulbecco's modified Eagle's medium (DMEM), fetal bovine serum (FBS), trypsin and penicillin was purchased from Gibco, United States. 


\subsection{Methods}

\subsubsection{Preparation of Niosomes}

TPGS-DTPA was synthesized and characterized following methods described previously (De Silva et al., 2019). Niosomes were prepared by simple sonication method as previously described by De Silva et al. (2019). Briefly, a mixture of Span 60, cholesterol, TPGS and synthesized TPGS-DTPA at molar ratio of 7.5:7.5:1:2 were vigorously stirred in PBS for $1 \mathrm{~h}$ at $60^{\circ} \mathrm{C}$. Sonication was performed using a Q125 sonicator (Qsonica L.L.C, Newton, CT, United States; $\max 125 \mathrm{~W}$ ) with the instrument set at $75 \%$ of its maximal capacity (measured power $94 \mathrm{~W}$ ) for $8 \mathrm{~min}$. Excess unbound materials were removed by size exclusion chromatography using Sephadex G-25.

\subsubsection{Particle Size and Zeta Potential Measurements}

The particle size diameter (nm) and polydispersity index (PDI) of the niosomes were measured in triplicates by cumulant analysis with DLS using the Zetasizer Nano ZS (Malvern Instruments, Malvern, United Kingdom). The analysis was performed using a $\mathrm{He}-\mathrm{Ne}$ laser (wavelength of $633 \mathrm{~nm}$ ) and a detector angle of $173^{\circ}$ (refractive index $=1.33$, viscosity $=0.8872 \mathrm{cP}$ ) at $25^{\circ} \mathrm{C}$. Samples were diluted with Milli-Q water (dilution factor 1:1,000) before analysis. Particle size diameter measurements data are expressed as mean $\pm \mathrm{SD}(\mathrm{nm})$.

The zeta potential $(\mathrm{mV})$ of the niosomes was measured in triplicates using Laser Doppler Electrophoresis on Zetasizer Nano ZS (Malvern Instruments). Samples were diluted with Milli-Q water (dilution factor 1:1,000) before analysis. Zeta potential measurements data are expressed as zeta potential $\pm \mathrm{SD}(\mathrm{mV})$.

\subsubsection{Morphology of Niosomes}

The niosomes were viewed using transmission electron microscope ([TEM], LIBRA 120; Carl Zeiss Meditec AG, Jena, Germany) operating at $120 \mathrm{kV}$. The niosomes were diluted with a Milli-Q water (dilution factor 1:10) before viewing. A drop of the diluted sample was then placed on a copper grid and allowed to air-dry at room temperature. Negative staining was performed with phosphotungstic acid. Any excess liquid was removed by drawing off with a piece of filter paper and left to dry in a desiccator. For viewing, the grid was mounted in the instrument and the electron images were captured under magnification of $\times 800$.

\subsubsection{Radiolabeling of Niosomes}

The niosomes were radiolabeled with $99 \mathrm{mTc}$ by surface chelation with TPGS-DTPA. $99 \mathrm{mTc}$-pertechnetate solution $\left(\mathrm{Na}^{99 \mathrm{~m}} \mathrm{TcO}_{4}{ }^{-}\right)$ was obtained by elution from a sterile $99 \mathrm{Mo} /{ }^{99 \mathrm{~m}} \mathrm{Tc}$ generator using $0.9 \% \mathrm{w} / \mathrm{v} \mathrm{NaCl}$ solution. Briefly, $1 \mathrm{ml}$ of $99 \mathrm{mTcO}_{4}{ }^{-}(2 \mathrm{mCi} /$ $\mathrm{ml}$ ) was mixed with $\mathrm{SnCl}_{2} \cdot \mathrm{H}_{2} \mathrm{O}$. The $\mathrm{pH}$ was adjusted to $\mathrm{pH} 5$ using $0.5 \mathrm{M} \mathrm{NaHCO}_{3}$ or $0.1 \mathrm{M} \mathrm{HCl}$ solution. To that mixture, $1 \mathrm{ml}$ of niosomal suspension was added and left to stand at room temperature for $15 \mathrm{~min}$.

\subsubsection{Radiolabeling Efficiency}

The percentage of radiolabeling efficiency of the radiolabeled complex was measured using methods described earlier [22]. The radiolabeling efficiency was estimated with ascending ITLC using
$1 \times 10 \mathrm{~cm} \mathrm{SG}$-coated fiber glass sheets (Agilent Technologies, Santa Clara, CA, United States) as the stationary phase. Approximately 2-3 $\mu \mathrm{l}$ of the radiolabeled complex was applied at a point $1 \mathrm{~cm}$ from one end of the ITLC-SG strip and allowed to dry at room temperature. The strips were allowed to develop for $8 \mathrm{~cm}$ from the point of application in appropriate solvent systems. ITLC was performed using two separate mobile phases.

During the radiolabeling process, three products were generated, namely $99 \mathrm{mTc}$-labeled niosomes, $99 \mathrm{mTcO}_{4}{ }^{-}$and radiocolloids. Saline was used to determine the percentage of $99 \mathrm{mTcO}_{4}{ }^{-}$that moved with the solvent front $\left(\mathrm{R}_{\mathrm{f}}=1.0\right)$, while radiocolloids and $99 \mathrm{mTc}$-labeled niosomes remained at the origin $\left(R_{f}=0\right)$. A separate mobile phase containing pyridine, acetic acid, and water was used to determine the amount of radiocolloids, where radiocolloids remained at the origin $\left(\mathrm{R}_{\mathrm{f}}=0\right)$ while $99 \mathrm{mTcO}_{4}{ }^{-}$and $99 \mathrm{mTc}$-labeled niosomes moved to the solvent front $\left(\mathrm{R}_{\mathrm{f}}=1.0\right)$.

After developing the strips in each mobile phase, the strips were dried and cut horizontally into two equal halves and the radioactivity (counts per minute (CPM)) in each segment was determined using an automated well-type gamma ray counter (2,470 Wizard ${ }^{2}$ Automatic Gamma Counter; PerkinElmer Inc, Waltham, MA, United States). The percentage of radiolabeling efficiency was determined by subtracting the sum of percentage of $99 \mathrm{mTcO}_{4}{ }^{-}$and radiocolloids from $100 \%$.

\subsubsection{In vitro Stability Study of 99mTc-Labeled Niosomes}

The in vitro stability of the $99 \mathrm{mTc}$-labeled niosomes was determined by ascending ITLC-SG technique. 99mTc-labeled niosomes $(0.1 \mathrm{ml})$ was incubated with $0.9 \%$ saline or mouse serum $(1.9 \mathrm{ml})$ at $37^{\circ} \mathrm{C}$ for up to $8 \mathrm{~h}$. After $0,1,3,6$ and $8 \mathrm{~h}$ of incubation, samples were drawn and ITLC was carried out to assess the percentage of dissociation or degradation of the 99mTc-labeled niosomes.

\subsubsection{Tumor Implantation}

All animal experiments conducted were in accordance to and approved by Universiti Kebangsaan Malaysia Animal Ethics Committee (MONASH/2016/ALICE/23-MAR./731-MARCH2016-DEC.-2018) for the purpose of control and supervision on experiments on animals. Female BALB/c mice (aged 4-6 weeks), weighing between 17 and $21 \mathrm{~g}$ were selected for this study. The mice were housed in a pathogen-free isolation facility under a $12 \mathrm{~h}$ light-dark cycle at $22-24^{\circ} \mathrm{C}$ and $50 \%$ humidity, and were fed ad libitum diet and water.

Mouse breast tumor cells 4T1 (ATCC, Manassas, VA, United States) were maintained in DMEM supplemented with $10 \%$ FBS and 100 units/mL penicillin/streptomycin. Cells were kept in a humidified atmosphere containing 5\% $\mathrm{CO}_{2}$ at $37^{\circ} \mathrm{C}$. For tumor inoculation, 4T1 cancer cells within $70-80 \%$ confluence were trypsinized, centrifuged $(5 \mathrm{~min}$ at $320 \mathrm{~g}$ ) and re-suspended in serum-free medium at a concentration of $1 \times 10^{7}$ cells $/ \mathrm{ml}$.

The mice were inoculated subcutaneously with 4 T 1 cells $(1 \times$ $10^{6}$ cells/mouse) on the right flank. Tumor growth was monitored daily and tumor size was measured with a digital caliper. Tumor 
volume (V) was calculated by using longitudinal cross section (L) and transverse section (W) as follows:

$$
\mathrm{V}\left(m m^{3}\right)=W^{2} \times \frac{L}{2}
$$

\subsubsection{Scintigraphic Imaging}

Tumor-bearing $\mathrm{BALB} / \mathrm{c}$ mice were used for scintigraphic imaging and biodistribution studies. When tumors reached a volume of $\sim 300 \mathrm{~mm}^{3}$, the mice $(n=3)$ were injected intravenously (tail vein) with $99 \mathrm{mTc}$-labeled niosomes $(200 \mu \mathrm{Ci}$; pH adjusted to 7 with 0.5 $\left.\mathrm{NaHCO}_{3}\right)$ or $99 \mathrm{mTcO}_{4}{ }^{-}(200 \mu \mathrm{Ci})$. At pre-determined time-points (1, 3, 6 and $8 \mathrm{~h}$ post injection), the mice were anesthetized with a mixture of ketamine/xylazine/Zoletil $50^{\circledR}$ for the acquisition of images. The mice were placed horizontally under the collimator of a gamma camera (BHP6602 T-Quest, Hamamatsu, China) and kept under anesthesia for all the duration of the imaging. Images were acquired using a 1,024 × 1,024 matrix size at $140 \mathrm{keV}$. Scans were carried out until 200,000 counts were obtained. Interpretation of the images were done visually.

\subsubsection{Biodistribution Studies}

Biodistribution studies were carried out with adaptions from a method by Arulsudar et al. (2003). When tumors reached a volume of $\sim 300 \mathrm{~mm}^{3}$, the mice were injected intravenously with $99 \mathrm{mTc}-$ labeled niosomes $\left(200 \mu \mathrm{Ci}\right.$; $\mathrm{pH}$ adjusted to 7 with $\left.0.5 \mathrm{NaHCO}_{3}\right)$. Mice $(n=5)$ were sacrificed at $1,3,6$ or $8 \mathrm{~h}$ post-injection; blood samples were immediately obtained by cardiac puncture. Whole liver, spleen, kidney, muscle, lungs, heart, stomach, intestines and tumor were all dissected and removed, washed with $0.9 \% \mathrm{w} / \mathrm{v}$ saline, dried and placed in pre-weighed plastic test tubes.

To correct for physical decay and to calculate the radiopharmaceutical uptake in each organ, a standard dosage containing suitably diluted aliquots (dilution factor of 50) of the injected solution was counted simultaneously in a separate tube at each time point. The samples were counted against $1 \mathrm{ml}$ suitable diluted aliquots of the injected solution. The radioactivity in each organ was counted using a well-type gamma counter and CPM values were calculated as the percentage injected dose per Gram of wet tissue. The data from the gamma counter was converted to percentage of injected dose (\%ID) by dividing the CPM in each tissue or organ with the total standard dose CPM and multiplying it with $100 \%$. The $\% \mathrm{ID} / \mathrm{g}$ of tissue or organ was obtained.

Formulae for calculation of \%ID/g of tissue or organ are stated below:

$$
\begin{gathered}
\text { Standard Dose }(S D)=\text { Standard count } \times 50(\text { dilution factor }) \\
\text { Injected Dose }(I D)=\text { Standard Dose }- \text { Tail Count } \\
\% \text { of ID in tissue or organ }=\frac{\text { Count in tissue or organ }}{I D} \times 100 \% \\
\% \text { of ID per gof tissue or organ }(\% \text { ID } / g) \\
=\frac{[\% \text { of injected dose in tissue or organ }]}{[\text { weight of tissue or organ }]}
\end{gathered}
$$

\subsubsection{Statistical Analysis}

All data are reported as mean \pm SD. The assessment of statistical significance among the results was carried out by Kruskal-Wallis followed by Dunnett's post hoc test using GraphPad PRISM 7.0 (GraphPad Software, Inc, La Jolla, CA, United States), and $p<$ 0.05 was considered statistically significant.

\section{RESULTS}

\subsection{Characterisation of Niosomes}

The formulated niosomes had an average particle size of $110.2 \pm$ $0.7 \mathrm{~nm}$ with a PDI $0.229 \pm 0.008$, indicating good homogeneity of the dispersion. The measured zeta potential showed that the niosomes were negatively charged, with an average value of $-64.8 \pm 1.2 \mathrm{mV}$, indicating that the formulated dispersion is stable. The radiolabeling efficiency of the $99 \mathrm{mTc}$-labeled niosomes was found to be $98.08 \%$. Figure 1 shows the TEM image of the niosomes taken under magnification of $\times 800$, and the size corroborates the data from zeta sizer.

\subsection{In vitro Stability Study of 99mTc-Labeled Niosomes}

The stability of $99 \mathrm{mTc}$-labeled niosomes was estimated by incubating the radiolabeled compound in saline and mouse serum at $37^{\circ} \mathrm{C}$ for pre-determined time intervals of up to $8 \mathrm{~h}$ (Figure 2). This is to mimic the condition in mice and to ensure that the radiolabeled compounds can withstand the in vivo condition before injection into the animals. The results revealed good stability of the $99 \mathrm{mTc}$-labeled niosomes in these conditions. The $99 \mathrm{mTc}-$ labeled niosomes showed no significant reductions in radiolabeling efficiency with a loss of 3.50 and $14.40 \%$ of radioactivity in saline and serum respectively after $8 \mathrm{~h}$, indicating that there was little dissociation of $99 \mathrm{mTc}$ and that the $99 \mathrm{mTc}$-labeled niosomes are suitable to be used for further in vivo studies. The values are in agreement with values reported in the literature on in vitro stability (De Barros et al., 2010; Eid Moustapha et al., 2016).

\subsection{Scintigraphic Imaging}

After injection into the animals, the biodistribution of free $99 \mathrm{mTcO}_{4}{ }^{-}$and $99 \mathrm{mTc}$-labeled niosomes in tumor-bearing mice was visualized by scintigraphic imaging. The images in Figure 3 show that $99 \mathrm{~m} \mathrm{TcO}_{4}{ }^{-}$accumulated predominantly in the stomach, intestines and thymus gland. In contrast, images of $99 \mathrm{mTc}$-labeled niosomes (Figure 4) showed accumulation predominantly in the liver and spleen with minimal to no radioactivity uptake in the stomach and thyroid, indicating minimal leaching of $99 \mathrm{mTcO}_{4}{ }^{-}$ from the radiolabeled complex in vivo. The differential distribution profiles of free $99 \mathrm{mTcO}_{4}{ }^{-}$and $99 \mathrm{mTc}$-labeled niosomes are demonstrated here and verifies that the results seen in Figure 4 are not due to detachment of free Tc.

\subsection{Biodistribution Studies}

Figure 5 shows the accumulation of $99 \mathrm{mTc}$-labeled niosomes in various organs after tail vein injection in tumor-bearing mice at various time intervals. After $1 \mathrm{~h}$, the niosomes were found to be 


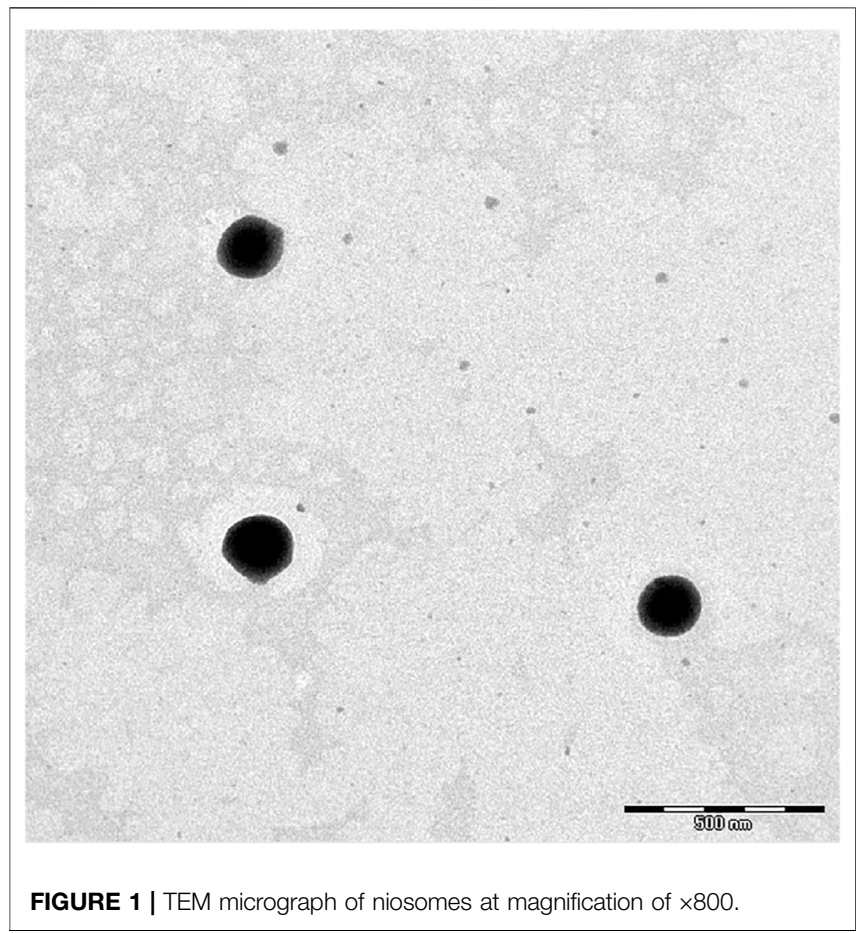

located primarily in the liver and spleen with an average $\%$ ID/g of $3.61 \pm 0.26$ and $4.96 \pm 0.84$, respectively. Mean liver uptake of $99 \mathrm{mTc}$-labeled niosomes dropped slightly to $3.39 \pm 0.61 \%$ at $3 \mathrm{~h}$, followed by an increase to $6.38 \pm 2.35 \%$ at $6 \mathrm{~h}$ and decreased once again to $3.02 \pm 0.74 \%$ at $8 \mathrm{~h}$. On the other hand, the mean uptake of the spleen showed an initial increase from $4.96 \pm 0.84$ at $1 \mathrm{~h}$ to $5.42 \pm 1.35 \%$ at $3 \mathrm{~h}$ followed by a gradual decrease to $4.78 \pm 1.53$ and $4.31 \pm 0.32 \%$ at 6 and $8 \mathrm{~h}$, respectively. Very little radioactivity was recovered in off-target organs and tissues such as the heart, lungs, stomach intestine and muscles. In the bloodstream, a peak concentration of $99 \mathrm{mTc}$-labeled niosomes with a $\% \mathrm{ID} / \mathrm{g}$ of $9.13 \pm 0.98$ was observed $1 \mathrm{~h}$ after injection. The levels of $99 \mathrm{mTc}$-labeled niosomes decreased over time, which can be attributed to the liver and spleen uptake followed by the renal elimination during the evaluated time points. The biodistribution of $99 \mathrm{mTc}$-labeled niosomes in the tumor showed an initial \% ID/ $\mathrm{g}$ of $1.01 \pm 0.18$ at $1 \mathrm{~h}$, followed by a steady decrease to $0.83 \pm 0.20$, $0.70 \pm 0.21$ and $0.35 \pm 0.07$ at 3,6 and 8 h, respectively. Figure 6 shows the tumor-to-muscle ratios achieved at 1, 3, 6 and $8 \mathrm{~h}$ after intravenous injections of $99 \mathrm{mTc}$-labeled niosomes in tumorbearing mice. The tumor-to-muscle ratio showed an increasing trend from $1.35 \pm 0.40$ after $1 \mathrm{~h}$, to $1.97 \pm 0.65$ after $3 \mathrm{~h}$, followed by a maximum of $4.48 \pm 2.73$ at $6 \mathrm{~h}$ and subsequently decreased to $3.57 \pm 1.53$ at $8 \mathrm{~h}$. However, no significant difference was detected between the time points due to high variability.

\section{DISCUSSION}

Previous literature has shown that nanocarriers with particle sizes ranging between $100-150 \mathrm{~nm}$ display improved accumulation within tumors in comparison to nanocarriers with particle sizes lower than $50 \mathrm{~nm}$ or greater than $300 \mathrm{~nm}$ (Allen et al., 2006; Li et al., 2008; Ferreira et al., 2012; Fanciullino et al., 2014). Furthermore, small particle sizes confer greater stability in the circulation by the avoiding the MPS system (Fanciullino et al., 2014). The absence of microvasculature occlusion or pulmonary embolism from the systemic administration of the formulated $99 \mathrm{mTc}$-labaled niosomes supports that the delivery system possesses suitable physical characteristics for in vivo use. The zeta potential obtained indicates strong repellant forces on the surface of the niosomes that can prevent aggregation (Patel et al., 2016).

TPGS is a non-ionic, amphiphilic molecule consisting of a lipophilic non-polar (water-insoluble) head and a hydrophilic (water-soluble) PEG tail and is formed through the esterification of D-alpha-tocopheryl acid succinate and PEG 1000 (Huang et al., 2011; Guo et al., 2013; Muthu et al., 2015; Patel et al., 2016). In an aqueous environment, the hydrophilic PEG tail becomes saturated with water molecules, thereby increasing the stability and increasing steric hindrance of TPGS coated structures (Muthu et al., 2015). It has also been reported that the size of niosomes can be reduced through the incorporation of TPGS, resulting in reduced bilayer defects in the niosomes, thereby improving the lateral packing of the acyl chains of the surfactant in the bilayer (Garbuzenko et al., 2005). In stability study, niosomes with TPGS remained stable up to 60 days as changes in size, polydispersity index and zeta potential were less than 17\% (Maniam et al., 2021).

The niosomes were radiolabeled by direct radiolabeling through the reduction of $99 \mathrm{mTcO}_{4}{ }^{-}$in the presence of $\mathrm{SnCl}_{2} \cdot \mathrm{H}_{2} \mathrm{O}$. In comparison, a study by Korkmaz et al. (2006) incorporated $99 \mathrm{mTc}$ TTPA into the niosome by thin film evaporation (Uchegbu, 2000). While they were able to similarly achieve high radiolabeling efficiency, the method involves higher radiation exposure to the personnel, as the niosomes are radiolabeled during the start of the manufacturing process. Additionally, the short half-life of $99 \mathrm{mTc}$ would require immediate administration into a patient after synthesis, and requiring substantial amount of work in a limited period of time. Scintigraphic imaging has been widely used to provide information on whole body distribution of radiolabeled compounds at different time intervals in a live animal or human subject (Bao et al., 2004; Monteiro et al., 2018). Figure 3 shows a series of anterior whole-body images of the distribution of $99 \mathrm{mTcO}_{4}{ }^{-}$in tumor-bearing mice captured at $1,3,6$, and $8 \mathrm{~h}$ post-injection. The images indicate that $99 \mathrm{mTcO}_{4}^{-}$predominantly accumulated in the thymus gland and digestive system, which is in good agreement with previously reported data on the distribution of $99 \mathrm{mTcO}_{4}{ }^{-}$in vivo (Valenca et al., 2005; Chacon et al., 2007). This accumulation occurs as the thymus gland and stomach are the main elimination organs of $99 \mathrm{mTcO}_{4}{ }^{-}$(Ferreira et al., 2015; Silva et al., 2016). In comparison, in Figure 4, the images of the mouse injected with 99mTc-labeled niosomes showed minimal radioactivity in the thymus gland and digestive system, indicating minimal dissociation and degradation of the radiolabeled complex in vivo. The high accumulation of $99 \mathrm{mTc}$-labeled niosomes in the liver and spleen at later time points can be attributed to 


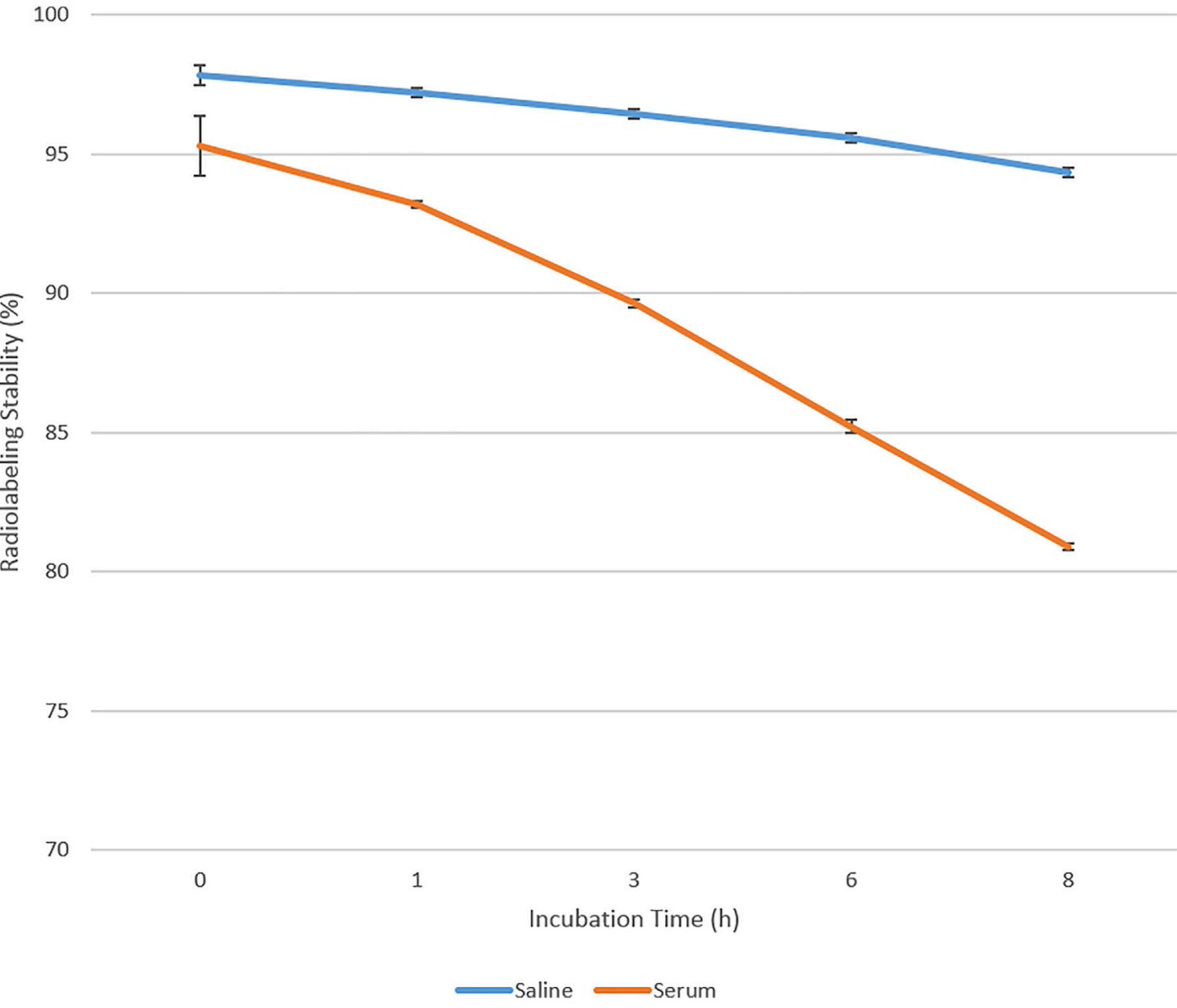

FIGURE 2 | In vitro stability of 99mTc-labeled niosomes.

1h

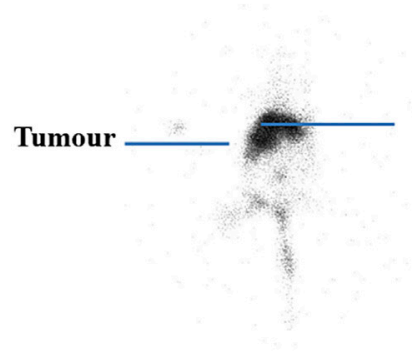

$3 \mathbf{h}$

$6 h$

$8 h$
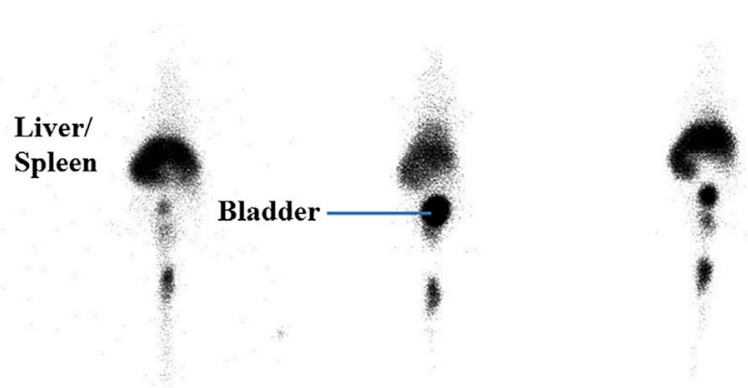

FIGURE 3 | Scintigraphic images acquired from a female nude BALB/c mice at 1, 3, 6 and $8 \mathrm{~h}$ post-injection with $200 \mu \mathrm{Ci} 99 \mathrm{mTcO}_{4}{ }^{-}$

the natural mechanism of vesicular clearance (Arulsudar et al., 2004; Gharepapagh et al., 2021). The planar images obtained from scintigraphic imaging provides qualitative information, as the activity in certain organs are seen to partially overlap (Fragogeorgi et al., 2014). For example, images of the lungs/ liver, liver/spleen and kidneys/intestines could be difficult to differentiate in the images. This data is therefore supplemented with quantification study.

Quantification of radioactivity in isolated organs and tissues provides an accurate and thorough understanding of the 

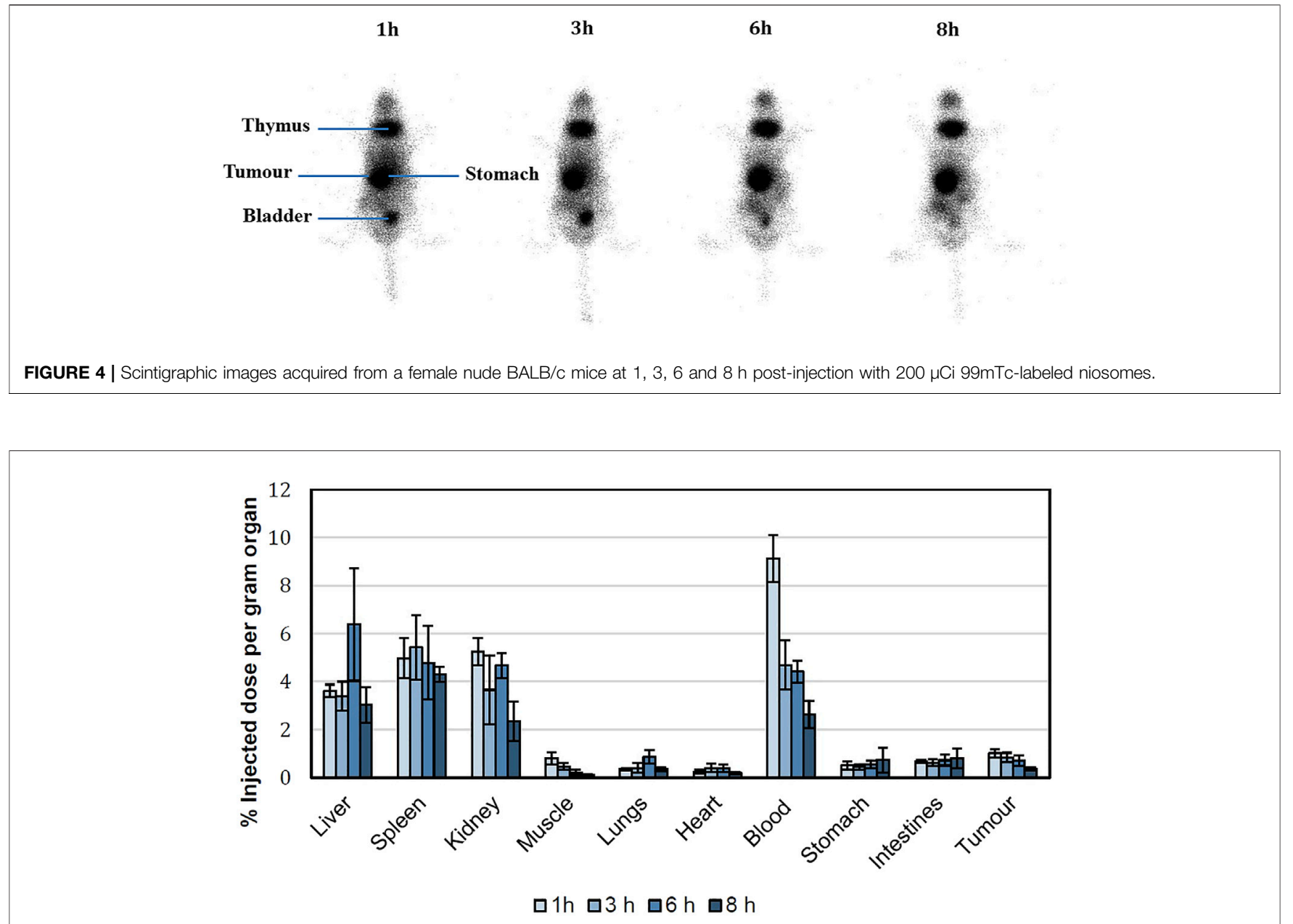

FIGURE 5 | Biodistribution of 99mTc-labeled niosomes in 4T1 tumor-beating female BALB/c mice expressed as \%ID/g per organ.

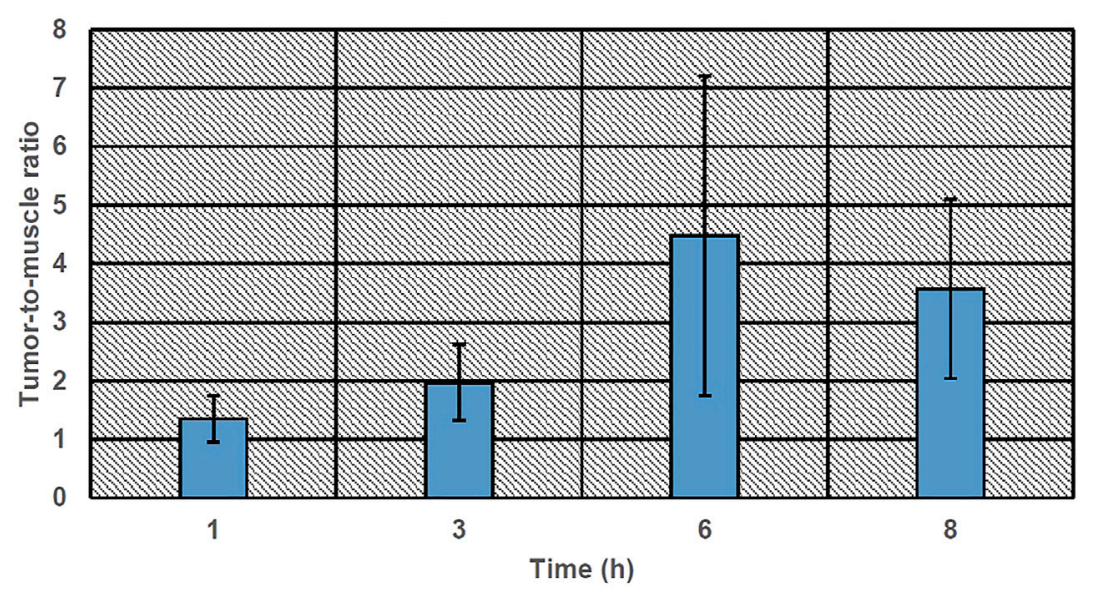

FIGURE 6 | Tumor-to-muscle ratio of 99mTc-labeled niosomes. 
biodistribution of $99 \mathrm{mTc}$-labeled niosomes. The results were in agreement with the biodistribution profiles seen in the scintigraphic images. Upon entering the circulation, there was considerable radioactivity (median $>3 \% \mathrm{ID} / \mathrm{g}$ ) in the clearance organs (liver, spleen and kidney). Vesicular systems are typically eliminated via phagocytosis, primarily by macrophages from the liver and spleen and consequently taken up by the reticuloendothelial system (RES). This effect has been similarly observed in previous studies with other radiolabeled niosomes (Almasi et al., 2018). In our study, the high \%-ID/g observed in the liver and spleen may be attributed to the interactions between the negatively charged groups on the surface of the niosomes and the cell surface proteins (Arulsudar et al., 2004). Juliano et al. (1975) and Dobrovolskaia et al. (2008) reported that negatively charged nanoparticles exhibit higher macrophage uptake in comparison to positively charged and neutral nanoparticles as a result of their tendency to coalesce in the presence of proteins and calcium ion in blood plasma (Juliano et al., 1975; Dobrovolskaia et al., 2008). However, this phenomenon was unavoidable in our formulation as the negative charge on the surface of our nanocarrier arises from the DTPA molecule, which is required for labeling $99 \mathrm{mTc}$ to the niosomes.

The ideal biodistribution pattern of $99 \mathrm{mTc}$-labeled niosomes would include little to no radioactivity in the thyroid gland, stomach and kidneys; indicating that the reduced $99 \mathrm{mTc}$ is tightly bound to the niosomes. Low radioactivity was detected in the stomach and intestines throughout the study with a maximum average $\% \mathrm{ID} / \mathrm{g}$ of $0.72 \pm 0.53$ and $0.80 \pm 0.41$, respectively at the $8 \mathrm{~h}$ time-point. The presence of a detectable amount of radioactivity noted in the stomach could be attributed to the self-grooming phenomenon that typically occurs in mice (Santini et al., 2017). The radioactivity found in the kidney following intravenous administration suggests that the $99 \mathrm{mTc}-$ labeled niosomes are excreted through the renal route upon splenic and hepatic metabolism (Kamal et al., 2018).

A good nanocarrier for anti-cancer drug delivery should possess a high capacity to target tumor tissues with minimal to low accumulation in off-target tissues to maximize tumor uptake and minimize toxicity to healthy tissues. In the tumor, the radioactivity was around $1 \% \mathrm{ID} / \mathrm{g}$, which was comparable to that of previously published studies (Richardson et al., 1977; Oku et al., 1993; Arulsudar et al., 2004; Fragogeorgi et al., 2014). While radioactivity at the tumor site compared to the total body activity was considerably low, there was a significant ratio of $99 \mathrm{mTc}$ labeled niosomes uptake in the tumor in comparison to the muscle. Accumulation of radioactivity is affected by the route of administration (Bahadori et al., 2021), where nearly perfect tumor retention was observed when radiolabelled nanoparticles are injected intratumorally (Moeendarbari et al., 2016). In this study, niosomes were injected intravenously, hence a lower overall accumulation should be expected. In fact, literature has reported that a tumor-to-muscle ratios of larger than 1.5 signifies that there is a $50 \%$ greater capture of the compound in the tumor tissue (Phillips et al., 1999; De Barros et al., 2010), which validates our results of tumor accumulation following intravenous injection. Figure 6 shows that $99 \mathrm{mTc}$-labeled niosomes had a tumor-to-muscle ratio of above 1.5 after $3 \mathrm{~h}$, indicating the ability of the niosomes to accumulate within the tumor microenvironment through EPR effect. Though no significant difference can be observed between the time points, the tumorto-muscle ratios are above 1.5 after $1 \mathrm{~h}$, showing preferential tumor accumulation. The EPR effect has been a leading factor in influencing nanocarrier formulation for anti-cancer therapy due to its proven efficacy in animal models. However, the heterogeneity of the EPR response in humans has raised questions regarding the universal validity of EPR among researchers (Danhier et al., 2016). A study with radiolabeled liposomes showed positive correlation between neoangiogenesis and EPR effect while interstitial fluid pressure at tumor microenvironment had little effect (Børresen et al., 2020). Having said that, there have been many successful developments of nanomedicines based around the EPR effect with excellent treatment efficacy over the years. A phase II trial of PEGlyated liposomal doxorubicin (DOXIL ${ }^{\circledR} /$ CAELYX $^{\circledR}$ ) showed that IV infusion of the nanoformulation resulted in anti-tumor activity with improved toxicity profile compared to standard IV bolus of doxorubicin in patients with soft tissue sarcoma, indicating tumor specificity (Judson et al., 2001). Recent studies have also found that the concurrent administration of vascular modulating agent can help overcome this through the selective passive opening of the defective endothelial cell gaps in tumor tissues (Nakamura et al., 2015).

The limitation of the current study includes the use of animal tumor model $4 \mathrm{~T} 1$ cells, in which all the data presented are on mice tumor. Future work could explore the use of human tumor cell lines implanted on nude mice. This would provide a tumor environment closer to human and generate added information prior to engaging in larger animal models or human studies. We also recognize that gamma scintigraphy is not the latest technology in animal imaging. More advanced imaging techniques, such a positron emitting tomography (PET) could produce images with higher resolution, but also at a higher cost. Moreover, the common radionuclide used in PET, Fluorine-18, has an extremely short halflife of $109.8 \mathrm{~min}$, which is not suitable for biodistribution studies involving longer time points. Thus, gamma scintigraphy presents as a cheaper and more accesible option, with its associated radionuclide $99 \mathrm{mTc}$ of $6 \mathrm{~h}$ half-life, making it a more suitable technique for our study. By utilizing this non-invasive imaging technique, we were able to avoid the unnecessary use of a large animal sample size before proceeding to a more comprehensive and invasive biodistribution study, where animals will be required to be sacrificed at each time-point (Bao et al., 2004).

\section{CONCLUSION}

In conclusion, biodistribution study of nanocarriers, such as niosomes, can be carried out non-invasively using scintigraphic imaging. We demonstrated successful prototype of niosomes conjugated with $99 \mathrm{mTc}$ through a simple and fast 1-step method. Based on the physical characteristics and stability features, our results indicate the feasibility of the developed niosomes for in vivo administration. With the high tumor to muscle uptake, the niosomes can act as a potential delivery system for improved tumor uptake of anti-cancer drugs. While 
controversy surrounds the validity of the EPR effect in humans, we maintain the belief that PEGylated niosome at $\sim 110 \mathrm{~nm}$ is a suitable candidate to achieve effective tumor penetration via the EPR effect. The niosomes formulated in this study offers promising results as a proof-of-concept and as a prototype for future development of niosomes for passive retention applications. Further enhancement can be achieved to augment the EPR effect such as alterations in blood flow and vascular permeability. The niosomes can be further designed to conjugate active targeting ligands to its surface, to improve the targeting ability and achieve even higher niosomes accumulation within the tumor microenvironment. This can then be used to encapsulate anticancer compounds for breast cancer targeting to achieve better treatment outcomes.

\section{DATA AVAILABILITY STATEMENT}

The original contributions presented in the study are included in the article/Supplementary Material, further inquiries can be directed to the corresponding author.

\section{REFERENCES}

Allen, T. M., Cheng, W. W., Hare, J. I., and Laginha, K. M. (2006). Pharmacokinetics and Pharmacodynamics of Lipidic Nano-Particles in Cancer. Anticancer Agents Med. Chem. 6 (6), 513-523. doi:10.2174/ 187152006778699121

Allen, T. M., and Cullis, P. R. (2013). Liposomal Drug Delivery Systems: from Concept to Clinical Applications. Adv. Drug Deliv. Rev. 65, 36-48. doi:10.1016/ j.addr.2012.09.037

Allen, T. M., Hansen, C., Martin, F., Redemann, C., and Yau-Young, A. (1991). Liposomes Containing Synthetic Lipid Derivatives of Poly(ethylene Glycol) Show Prolonged Circulation Half-Lives In Vivo. Biochim. Biophys. Acta 1066 (1), 29-36. doi:10.1016/0005-2736(91)90246-5

Almasi, A., Shahhosseini, S., Haeri, A., Daha, F. J., Geramifar, P., and Dadashzadeh, S. (2018). Radiolabeling of Preformed Niosomes with [99mTc]: In Vitro Stability, Biodistribution, and In Vivo Performance. AAPS PharmSciTech 19 (8), 3859-3870. doi:10.1208/s12249-018-1182-1

Amiri, B., Ahmadvand, H., Farhadi, A., Najmafshar, A., Chiani, M., and Norouzian, D. (2018). Delivery of Vinblastine-Containing Niosomes Results in Potent In Vitro/In Vivo Cytotoxicity on Tumor Cells. Drug Dev. Ind. Pharm. 44 (8), 1371-1376. doi:10.1080/03639045.2018.1451880

Arulsudar, N., Subramanian, N., Mishra, P., Chuttani, K., Sharma, R. K., and Murthy, R. S. (2004). Preparation, Characterization, and Biodistribution Study of Technetium-99m -labeled Leuprolide Acetate-Loaded Liposomes in Ehrlich Ascites Tumor-Bearing Mice. AAPS PharmSci 6 (1), E5. doi:10.1208/ps060105

Arulsudar, N., Subramanian, N., Mishra, P., Sharma, R. K., and Murthy, R. S. (2003). Preparation, Characterisation and Biodistribution of 99mTc-Labeled Liposome Encapsulated Cyclosporine. J. Drug Target. 11 (3), 187-196. doi:10.1080/10611860310001615415

Baban, D. F., and Seymour, L. W. (1998). Control of Tumour Vascular Permeability. Adv. Drug Deliv. Rev. 34 (1), 109-119. doi:10.1016/s0169409x(98)00003-9

Bahadori, S. R., Mulgaonkar, A. H. R., Wu, C-Y., Zhang, D., Pillai, A., Hao, Y., et al. (2021). Radiolabeling Strategies and Pharmacokinetic Studies for Metal Based Nanotheranostics. WIRES Nanomed. Nanobiotechnol. 13, e1671. doi:10.1002/ wnan. 1671

Bao, A., Goins, B., Klipper, R., Negrete, G., and Phillips, W. T. (2004). Direct $99 \mathrm{mTc}$ Labeling of Pegylated Liposomal Doxorubicin (Doxil) for Pharmacokinetic and Non-invasive Imaging Studies. J. Pharmacol. Exp. Ther. 308 (2), 419-425. doi:10.1124/jpet.103.059535

\section{ETHICS STATEMENT}

The animal study was reviewed and approved by the Universiti Kebangsaan Malaysia Animal Ethics Committee.

\section{AUTHOR CONTRIBUTIONS}

Conceptualization, writing-review and editing, funding acquisition, L-HC and J-YF; methodology, investigation, data curation, $\mathrm{LD}, \mathrm{TH}, \mathrm{WB}, \mathrm{AK}$, and $\mathrm{SM}$; writing-original draft preparation, LD; . All authors have read and agreed to the published version of the manuscript.

\section{FUNDING}

This research was funded by the Fundamental Research Grant Scheme (FRGS), by the Ministry of Higher Education, Malaysia (FRGS/2/2014/SG05/MUSM/03/3), and the Malaysian Palm Oil Board (MPOB).

Björnmalm, M., Thurecht, K. J., Michael, M., Scott, A. M., and Caruso, F. (2017). Bridging Bio-Nano Science and Cancer Nanomedicine. ACS Nano 11 (10), 9594-9613. doi:10.1021/acsnano.7b04855

Boerman, O. C., Laverman, P., Oyen, W. J., Corstens, F. H., and Storm, G. (2000). Radiolabeled Liposomes for Scintigraphic Imaging. Prog. Lipid Res. 39 (5), 461-475. doi:10.1016/s0163-7827(00)00013-8

Børresen, B., Hansen, A. E., Fliedner, F. P., Henriksen, J. R., Elema, D. R., BrandtLarsen, M., et al. (2020). Noninvasive Molecular Imaging of the Enhanced Permeability and Retention Effect by 64Cu-Liposomes: In Vivo Correlations with 68Ga-RGD, Fluid Pressure, Diffusivity and 18F-FDG. Int. J. Nanomedicine 15, 8571-8581. doi:10.2147/IJN.S239172

Byrne, J. D., Betancourt, T., and Brannon-Peppas, L. (2008). Active Targeting Schemes for Nanoparticle Systems in Cancer Therapeutics. Adv. Drug Deliv. Rev. 60 (15), 1615-1626. doi:10.1016/j.addr.2008.08.005

Chacon, D. D. A., Araújo-Filho, I., Villarim-Neto, A., Rêgo, A. C., Azevedo, I. M., Bernardo-Filho, M., et al. (2007). Biodistribution of the Radiophamarceutical Sodium Pertechnetate (Na99mTcO4) after Massive Small Bowel Resection in Rats. Acta Cir. Bras. 22 (6), 430-435. doi:10.1590/ s0102-86502007000600003

Chapman, S., Dobrovolskaia, M., Farahani, K., Goodwin, A., Joshi, A., Lee, H., et al. (2013). Nanoparticles for Cancer Imaging: The Good, the Bad, and the Promise. Nano Today 8 (5), 454-460. doi:10.1016/j.nantod.2013.06.001

Choudhury, H., Pandey, M., Chin, P. X., Phang, Y. L., Cheah, J. Y., Ooi, S. C., et al. (2018). Transferrin Receptors-Targeting Nanocarriers for Efficient Targeted Delivery and Transcytosis of Drugs into the Brain Tumors: a Review of Recent Advancements and Emerging Trends. Drug Deliv. Transl. Res. 8 (5), 1545-1563. doi:10.1007/s13346-018-0552-2

Danhier, F. (2016). To Exploit the Tumor Microenvironment: Since the EPR Effect Fails in the Clinic, what Is the Future of Nanomedicine? J. Control. Release 244, 108-121. doi:10.1016/j.jconrel.2016.11.015

De Barros, A. L., Cardoso, V. N., Mota, L. D., Leite, E. A., De Oliveira, M. C., and Alves, R. J. (2010). A Novel D-Glucose Derivative Radiolabeled with Technetium-99m: Synthesis, Biodistribution Studies and Scintigraphic Images in an Experimental Model of Ehrlich Tumor. Bioorg. Med. Chem. Lett. 20 (8), 2478-2480. doi:10.1016/j.bmcl.2010.03.003

De Silva, L., Fu, J. Y., Htar, T. T., Muniyandy, S., Kasbollah, A., Wan Kamal, W. H. B., et al. (2019). Characterization, Optimization, and In Vitro Evaluation of Technetium-99m-Labeled Niosomes. Int. J. Nanomedicine 14, 1101-1117. doi:10.2147/IJN.S184912

Ding, H., and Wu, F. (2012). Image Guided Biodistribution and Pharmacokinetic Studies of Theranostics. Theranostics 2 (11), 1040-1053. doi:10.7150/thno.4652 
Dobrovolskaia, M. A., Aggarwal, P., Hall, J. B., and Mcneil, S. E. (2008). Preclinical Studies to Understand Nanoparticle Interaction with the Immune System and its Potential Effects on Nanoparticle Biodistribution. Mol. Pharm. 5 (4), 487-495. doi:10.1021/mp800032f

Eid Moustapha, M., Shweeta, H. A., and Motaleb, M. A. (2016). Technetiumlabeled Danofloxacin Complex as a Model for Infection Imaging. Arabian J. Chem. 9, S1928-S1934. doi:10.1016/j.arabjc.2014.10.017

Etheridge, M. L., Campbell, S. A., Erdman, A. G., Haynes, C. L., Wolf, S. M., and Mccullough, J. (2013). The Big Picture on Nanomedicine: the State of Investigational and Approved Nanomedicine Products. Nanomedicine 9 (1), 1-14. doi:10.1016/j.nano.2012.05.013

Fanciullino, R., Mollard, S., Correard, F., Giacometti, S., Serdjebi, C., Iliadis, A., et al. (2014). Biodistribution, Tumor Uptake and Efficacy of 5-FU-Loaded Liposomes: Why Size Matters. Pharm. Res. 31 (10), 2677-2684. doi:10.1007/ s11095-014-1364-9

Ferreira, D. D. S., Boratto, F. A., Cardoso, V. N., Serakides, R., Fernandes, S. O., Ferreira, L. A., et al. (2015). Alendronate-coated Long-Circulating Liposomes Containing 99mtechnetium-Ceftizoxime Used to Identify Osteomyelitis. Int. J. Nanomedicine 10, 2441-2450. doi:10.2147/IJN.S76168

Ferreira, S. M., Domingos, G. P., Ferreira, D. D. S., Rocha, T. G., Serakides, R., de Faria Rezende, C. M., et al. (2012). Technetium-99m-labeled Ceftizoxime Loaded Long-Circulating and $\mathrm{pH}$-Sensitive Liposomes Used to Identify Osteomyelitis. Bioorg. Med. Chem. Lett. 22 (14), 4605-4608. doi:10.1016/ j.bmcl.2012.05.105

Fragogeorgi, E. A., Savina, I. N., Tsotakos, T., Efthimiadou, E., Xanthopoulos, S., Palamaris, L., et al. (2014). Comparative In Vitro Stability and Scintigraphic Imaging for Trafficking and Tumor Targeting of a Directly and a Novel ${ }^{99} \mathrm{mTc}(\mathrm{I})(\mathrm{CO})_{3}$ Labeled Liposome. Int. J. Pharm. 465 (1), 333-346. doi:10.1016/j.ijpharm.2014.01.042

Gao, X., Guo, L., Li, J., Thu, H. E., and Hussain, Z. (2018). Nanomedicines Guided Nanoimaging Probes and Nanotherapeutics for Early Detection of Lung Cancer and Abolishing Pulmonary Metastasis: Critical Appraisal of Newer Developments and Challenges to Clinical Transition. J. Control. Release 292 (292), 29-57. doi:10.1016/j.jconrel.2018.10.024

Garbuzenko, O., Barenholz, Y., and Priev, A. (2005). Effect of Grafted PEG on Liposome Size and on Compressibility and Packing of Lipid Bilayer. Chem. Phys. Lipids 135 (2), 117-129. doi:10.1016/j.chemphyslip.2005.02.003

Gharepapagh, E., Fakhari, A., Firuzyar, T., Shomali, A., and Azimi, F. (2021). Preparation, Biodistribution and Dosimetry Study of Tc-99m Labeled N-Doped Graphene Quantum Dot Nanoparticles as a Multimodular Radiolabeling Agent. New J. Chem. 45, 3909-3919. doi:10.1039/d0nj04762g

Goldman, E., Zinger, A., Da Silva, D., Yaari, Z., Kajal, A., Vardi-Oknin, D., et al. (2017). Nanoparticles Target Early-Stage Breast Cancer Metastasis In Vivo. Nanotechnology 28 (43), 43LT01. doi:10.1088/1361-6528/aa8a3d

Guo, Y., Luo, J., Tan, S., Otieno, B. O., and Zhang, Z. (2013). The Applications of Vitamin E TPGS in Drug Delivery. Eur. J. Pharm. Sci. 49 (2), 175-186. doi:10.1016/j.ejps.2013.02.006

Hafner, A., Lovrić, J., Lakoš, G. P., and Pepić, I. (2014). Nanotherapeutics in the EU: an Overview on Current State and Future Directions. Int. J. Nanomedicine 9, 1005-1023. doi:10.2147/IJN.S55359

Hak, S., Reitan, N. K., Haraldseth, O., and De Lange Davies, C. (2010). Intravital Microscopy in Window chambers: a Unique Tool to Study Tumor Angiogenesis and Delivery of Nanoparticles. Angiogenesis 13 (2), 113-130. doi:10.1007/s10456-010-9176-y

Haley, B., and Frenkel, E. (2008). Nanoparticles for Drug Delivery in Cancer Treatment. Urol. Oncol. 26 (1), 57-64. doi:10.1016/j.urolonc.2007.03.015

Hatakeyama, H., Akita, H., and Harashima, H. (2011). A Multifunctional Envelope Type Nano Device (MEND) for Gene Delivery to Tumours Based on the EPR Effect: A Strategy for Overcoming the PEG Dilemma. Adv. Drug Deliv. Rev. 63 (3), 152-160. doi:10.1016/j.addr.2010.09.001

He, B., Sui, X., Yu, B., Wang, S., Shen, Y., and Cong, H. (2020). Recent Advances in Drug Delivery Systems for Enhancing Drug Penetration into Tumors. Drug Deliv. 27 (1), 1474-1490. doi:10.1080/10717544.2020.1831106

Huang, L., Chen, H., Zheng, Y., Song, X., Liu, R., Liu, K., et al. (2011). Nanoformulation of D-a-Tocopheryl Polyethylene Glycol 1000 SuccinateB-Poly( $\varepsilon$-Caprolactone-Ran-Glycolide) Diblock Copolymer for Breast Cancer Therapy. Integr. Biol. (Camb) 3 (10), 993-1002. doi:10.1039/ clib00026h
Hussain, Z., Khan, S., Imran, M., Sohail, M., Shah, S. W. A., and de Matas, M. (2019). PEGylation: a Promising Strategy to Overcome Challenges to CancerTargeted Nanomedicines: a Review of Challenges to Clinical Transition and Promising Resolution. Drug Deliv. Transl Res. 9 (3), 721-734. doi:10.1007/ s13346-019-00631-4

Hussain, Z., Rahin, M. A., Jan, N., Shah, H., Rawas-Qalaji, M., Khan, S., et al. (2021). Cell Membrane Cloaked Nanomedicines for Bio-Imaging and Immunotherapy of Cancer: Improved Pharmacokinetics, Cell Internalization and Anticancer Efficacy. J. Control. Release 292, 29-57. doi:10.1016/ j.jconrel.2021.05.018

Jackson, P. A., Hofman, M. S., Hicks, R. J., Scalzo, M., and Violet, J. (2020). Radiation Dosimetry in 177Lu-PSMA-617 Therapy Using a Single Posttreatment SPECT/CT Scan: A Novel Methodology to Generate Timeand Tissue-specific Dose Factors. J. Nucl. Med. 61 (7), 1030-1036. doi:10.2967/ jnumed.119.233411

Judson, I., Radford, J. A., Harris, M., Blay, J. Y., van Hoesel, Q., le Cesne, A., et al. (2001). Randomised Phase II Trial of Pegylated Liposomal Doxorubicin (DOXIL/CAELYX) versus Doxorubicin in the Treatment of Advanced or Metastatic Soft Tissue Sarcoma: a Study by the EORTC Soft Tissue and Bone Sarcoma Group. Eur. J. Cancer 37 (7), 870-877. doi:10.1016/s09598049(01)00050-8

Juliano, R. L., and Stamp, D. (1975). The Effect of Particle Size and Charge on the Clearance Rates of Liposomes and Liposome Encapsulated Drugs. Biochem. Biophys. Res. Commun. 63 (3), 651-658. doi:10.1016/s0006-291x(75)80433-5

Kamal, R., Chadha, V. D., and Dhawan, D. K. (2018). Physiological Uptake and Retention of Radiolabeled Resveratrol Loaded Gold Nanoparticles (99mTcRes-AuNP) in colon Cancer Tissue. Nanomedicine 14 (3), 1059-1071. doi:10.1016/j.nano.2018.01.008

Korkmaz, M., and Ozer, Y. A. (2006). DTPA Liposomes in Diagnostic Imaging. Fabad J. Pharm. Sci. 31 (4), 210-218. http://dergi.fabad.org.tr/pdf/volum31/ issue4/210-219.pdf

Li, S. D., and Huang, L. (2008). Pharmacokinetics and Biodistribution of Nanoparticles. Mol. Pharm. 5 (4), 496-504. doi:10.1021/mp800049w

Maeda, H. (2021). The 35th Anniversary of the Discovery of EPR Effect: A New Wave of Nanomedicines for Tumor-Targeted Drug Delivery-Personal Remarks and Future Prospects. J. Pers. Med. 11 (3), 229. doi:10.3390/jpm11030229

Maniam, G., Mai, C. W., Zulkefeli, M., and Fu, J. Y. (2021). Co-encapsulation of Gemcitabine and Tocotrienols in Nanovesicles Enhanced Efficacy in Pancreatic Cancer. Nanomedicine (Lond) 16 (5), 373-389. doi:10.2217/nnm-2020-0374

Moeendarbari, S., Tekade, R., Mulgaonkar, A., Christensen, P., Ramezani, S., Hassan, G., et al. (2016). Theranostic Nanoseeds for Efficacious Internal Radiation Therapy of Unresectable Solid Tumors. Sci. Rep. 6, 20614. doi:10.1038/srep20614

Monteiro, L. O. F., Fernandes, R. S., Oda, C. M. R., Lopes, S. C., Townsend, D. M., Cardoso, V. N., et al. (2018). Paclitaxel-loaded Folate-Coated Long Circulating and pH-Sensitive Liposomes as a Potential Drug Delivery System: A Biodistribution Study. Biomed. Pharmacother. 97, 489-495. doi:10.1016/ j.biopha.2017.10.135

Muthu, M. S., Kulkarni, S. A., Xiong, J., and Feng, S. S. (2011). Vitamin E TPGS Coated Liposomes Enhanced Cellular Uptake and Cytotoxicity of Docetaxel in Brain Cancer Cells. Int. J. Pharm. 421 (2), 332-340. doi:10.1016/ j.ijpharm.2011.09.045

Muthu, M. S., Kutty, R. V., Luo, Z., Xie, J., and Feng, S. S. (2015). Theranostic Vitamin E TPGS Micelles of Transferrin Conjugation for Targeted Co-delivery of Docetaxel and Ultra Bright Gold Nanoclusters. Biomaterials 39, 234-248. doi:10.1016/j.biomaterials.2014.11.008

Nakamura, H., Fang, J., Jun, F., and Maeda, H. (2015). Development of NextGeneration Macromolecular Drugs Based on the EPR Effect: Challenges and Pitfalls. Expert Opin. Drug Deliv. 12 (1), 53-64. doi:10.1517/ 17425247.2014.955011

Nel, A., Ruoslahti, E., and Meng, H. (2017). New Insights into "Permeability" as in the Enhanced Permeability and Retention Effect of Cancer Nanotherapeutics. ACS Nano 11 (10), 9567-9569. doi:10.1021/acsnano.7b07214

Oku, N., Namba, Y., Takeda, A., and Okada, S. (1993). Tumor Imaging with Technetium-99m-DTPA Encapsulated in RES-Avoiding Liposomes. Nucl. Med. Biol. 20 (4), 407-412. doi:10.1016/0969-8051(93)90071-2

Patel, A., Tyagi, A., Sharma, R. K., and Thakkar, H. (2016). A Gamma Scintigraphy Study to Investigate Uterine Targeting Efficiency of Raloxifene-Loaded 
Liposomes Administered Intravaginally in New Zealand white Female Rabbits. Drug Deliv. 23 (9), 3330-3338. doi:10.1080/10717544.2016.1177137

Phillips, W. T. (1999). Delivery of Gamma-Imaging Agents by Liposomes. Adv. Drug Deliv. Rev. 37 (1), 13-32. doi:10.1016/s0169-409x(98)00108-2

Richardson, V. J., Jeyasingh, K., Jewkes, R. F., Ryman, B. E., and Tattersall, M. H. (1977). Properties of [99mTc] Technetium-Labelled Liposomes in Normal and Tumour-Bearing Rats. Biochem. Soc. Trans. 5 (1), 290-291. doi:10.1042/ bst0050290

Santini, C., Arranja, A. G., Denkova, A. G., Schosseler, F., Morawska, K., Dubruel, P., et al. (2017). Intravenous and Intratumoral Injection of Pluronic P94: The Effect of Administration Route on Biodistribution and Tumor Retention. Nanomedicine 13 (7), 2179-2188. doi:10.1016/j.nano.2017.04.015

Shaker, D. S., Shaker, M. A., and Hanafy, M. S. (2015). Cellular Uptake, Cytotoxicity and In-Vivo Evaluation of Tamoxifen Citrate Loaded Niosomes. Int. J. Pharm. 493 (1-2), 285-294. doi:10.1016/ j.ijpharm.2015.07.041

Shi, Y., van der Meel, R., Chen, X., and Lammers, T. (2020). The EPR Effect and beyond: Strategies to Improve Tumor Targeting and Cancer Nanomedicine Treatment Efficacy. Theranostics 10 (17), 7921-7924. doi:10.7150/thno.49577

Silva, J. O., Fernandes, R. S., Lopes, S. C., Cardoso, V. N., Leite, E. A., Cassali, G. D., et al. (2016). pH-Sensitive, Long-Circulating Liposomes as an Alternative Tool to Deliver Doxorubicin into Tumors: a Feasibility Animal Study. Mol. Imaging Biol. 18 (6), 898-904. doi:10.1007/s11307-016-0964-7

Subhan, M. A., Yalamarty, S. S. K., Filipczak, N., Parveen, F., and Torchilin, V. P. (2021). Recent Advances in Tumor Targeting via EPR Effect for Cancer Treatment. J. Pers Med. 11 (6), 571. doi:10.3390/jpm11060571
Uchegbu, I. F. (2000). Synthetic Surfactant Vesicles: Niosomes and Other Nonphospholipid Vesicular Systems. Amsterdam: Harwood Academic Abingdon.

Valenca, S. S., Lima, E. A., Dire, G. F., Bernardo-Filho, M., and Porto, L. C. (2005) Sodium Pertechnetate (Na99mTcO4) Biodistribution in Mice Exposed to Cigarette Smoke. BMC Nucl. Med. 5 (1), 1. doi:10.1186/1471-2385-5-1

Zhang, J., Zhang, J., Xu, X., Lu, L., Hu, S., Liu, C., et al. (2020). Evaluation of Radiation Dosimetry of 99mTc-HYNIC-PSMA and Imaging in Prostate Cancer. Sci. Rep. 10, 4179. doi:10.1038/s41598-020-61129-5

Conflict of Interest: The authors declare that the research was conducted in the absence of any commercial or financial relationships that could be construed as a potential conflict of interest.

Publisher's Note: All claims expressed in this article are solely those of the authors and do not necessarily represent those of their affiliated organizations, or those of the publisher, the editors, and the reviewers. Any product that may be evaluated in this article, or claim that may be made by its manufacturer, is not guaranteed or endorsed by the publisher.

Copyright (c) 2022 De Silva, Fu, Htar, Wan Kamal, Kasbollah, Muniyandy and Chuah. This is an open-access article distributed under the terms of the Creative Commons Attribution License (CC BY). The use, distribution or reproduction in other forums is permitted, provided the original author(s) and the copyright owner(s) are credited and that the original publication in this journal is cited, in accordance with accepted academic practice. No use, distribution or reproduction is permitted which does not comply with these terms. 